PROCEEDINGS OF THE AMERICAN MATHEMATICAL SOCIETY

Volume 124, Number 1, January 1996

\title{
ON LINEAR SERIES ON GENERAL $k$-GONAL PROJECTIVE CURVES
}

\author{
E. BALLICO AND C. KEEM
}

(Communicated by Eric M. Friedlander)

\begin{abstract}
Let $X$ be a general $k$-gonal curve of genus $g$. Here we prove a strong upper bound for the dimension of linear series on $X$, i.e. we prove that $\operatorname{dim}\left(W_{d}^{r}(X)\right) \leq \rho(g, r, d)+(g-2 k+2):=g-(r+1)(r+g-d)+(g-2 k+2)$.
\end{abstract}

In recent years several papers were devoted to the study of linear series on a general $k$-gonal curve. This note contains the proof of the following result.

Theorem 0.1. Fix integers $g, r, d, k$ with $k \geq 2,2 k-2 \leq g \leq 4 k-4,2 \leq r \leq d \leq$ $g-1$. Assume $\operatorname{char}(\mathbf{K})=0$ or $\operatorname{char}(\mathbf{K})>2 g$. Let $X$ be a general $k$-gonal curve of genus $g$. Then

$$
\operatorname{dim}\left(W_{d}^{r}(X)\right) \leq \rho(g, r, d)+(g-2 k+2):=g-(r+1)(r+g-d)+(g-2 k+2) .
$$

Here $\rho(g, r, d)=g-(r+1)(r+d-g)=(r+1)(d+r)-r g$ is the BrillNoether number. Note that the bound in the statement of 0.1 is just $\rho(2 k-2, r, d)-$ $(r-1)(g-2 k+2)=(r+1)(d+r)-r(2 k-2)-(r-1)(g-2 k-2)$ and this is a hint of the way in which we will prove the theorem in section 1 by induction on $g$ starting from the case $g=2 k-2$ (which is known to be true because a general curve of genus $2 k-2$ is a $k$-gonal curve). The tools are the theory of limit linear series of Eisenbud and Harris (see [EH2]) and the theory of admissible coverings of Harris-Mumford (see $[\mathrm{HM}]$ ). The reader is assumed to have a working knowledge of these theories. In this paper we work always over an algebraically closed base field $\mathbf{K}$ with either $\operatorname{char}(\mathbf{K})=0$ or $\operatorname{char}(\mathbf{K})>2 g$.

We believe that Theorem 0.1 is quite good for high $r$, but not sharp. For instance, it says nothing if $r=1$, while much is known about the pencils on $X$. The upper bound for $\operatorname{dim}\left(W_{d}^{r}(X)\right)$ in its statement just comes from part (d) of its proof.

Proof of 0.1 . The proof of Theorem 0.1 is divided into 4 steps.

Received by the editors May 18, 1994.

1991 Mathematics Subject Classification. Primary 14C95, 14C20.

Key words and phrases. Linear series, gonality, reducible algebraic curves, limit linear series.

The first author was partially supported by MURST and GNSAGA of CNR (Italy). He wants to thank GARC-KOSEF (Korea) and his mathematical Korean friends both for the mathematics and the hospitality. The second author was partially supported by MOE (Korea). Both authors are indebted to GARC-KOSEF at Seoul National University, since this note owes its existence to its warm and stimulating atmosphere.

(C)1996 American Mathematical Society 
(a) The assumption on $\operatorname{char}(\mathbf{K})$ allows us to use freely in the range of integers that we will consider the results on admissible coverings in $[\mathrm{HM}]$ and limit linear series in [EH1], [EH2], [EH3] and [EH4].

(b) Set $m:=2 k-2, s:=g-m$, and let $D$ be a general curve of genus $m$. By the generality of $D, D$ satisfies the Brill-Noether conditions, has the expected number of $g_{k}^{1}$ and each of them has $6 k-6$ simple ramification points. Fix one $g_{k}^{1}$ on $D$ and $s$ of its ramification points, $P_{1}, \ldots, P_{s}$. Let $A$ be the stable genus $g$ curve of compact type union of $D$ and $s$ disjoint elliptic curves $E_{1}, \ldots, E_{s}$, with each $E_{i}$ intersecting $D$ at $P_{i}$.

(c) First we will check that $A$ is a limit in $\bar{M}_{g}$ of smooth $k$-gonal curves of genus $g$. Indeed this curve is the stable reduction of an admissible degree $k$ covering $f: X^{\prime} \rightarrow U^{\prime}$ (in the sense of Harris-Mumford [HM]) (or see [EH2], sect. 5) with the following description. $U^{\prime}$ is a connected nodal curve with $p_{a}\left(U^{\prime}\right)=0 . X^{\prime}$ is the union of $D$ and $s(k-2) \mathbf{P}^{1}$ 's. The morphism $f$ sends $D$ to one of the $\mathbf{P}^{1}$ 's, say $J$, of $U^{\prime}$ as the chosen $g_{k}^{1}$; each of the $s$ families of $k-2 \mathbf{P}^{1}$ 's of $X^{\prime}$ are linked to $D$ at the other points of the $g_{k}^{1}$ over the image of $D \cap E_{i}$ and mapped by $f$ to a different component, say $J_{i}$, of $U^{\prime}$ with $u\left(\operatorname{Sing}\left(X^{\prime}\right)\right)=\operatorname{Sing}\left(U^{\prime}\right)$.

(d) To prove Theorem 0.1 it is sufficient to prove that the dimension of limit linear series (in the sense of Eisenbud-Harris) is bounded by $\rho(g, r, d)+s$ (and that the same is true for any curve which is obtained from $A$ by inserting chains of $\mathbf{P}^{1}$ s at each point of $\operatorname{Sing}(A)$ ). This (and all the Brill-Noether-Gieseker package) would hold if we had chosen general points of $D$ as $\operatorname{Sing}(A)$ instead of the points $P_{i}$; this was remarked (more or less explicitly) several times (see [EH3], Theorem 1.1, or [EH4], Proposition 5.2 and Corollary 5.3, or [W], Remarks 1.12 and 2.9). But of course, since $D$ has only finitely many $g_{k}^{1}$, the set $\left\{P_{i}\right\}_{1 \leq i \leq s}$ is not a general set of $s$ points of $D$. It is a standard calculation in the theory of limit linear series that each general point of $D$ to which an elliptic tail is linked "drops" the dimension of the set of all limits $g_{d}^{r}$ on $D$ (which have dimension $\rho(m, r, d)$ ) by $r$. We want to check that each $E_{i}$ imposes at least $r-1$ independent conditions to the set of all limits $g_{d}^{r}$ on $D$, proving 0.1 . Indeed we claim that this holds for any choice of the points $P_{i}$. To prove the claim we degenerate $D$ to the union of a general curve $Z$ of genus $m-s$ (which by assumption is $\geq 0$ ) and $s$ elliptic curves $F_{i}$ with $E_{i}$ linked to $F_{i}$. By the theory of admissible coverings of Harris-Mumford, to preserve the condition that the arithmetic genus $g$ curve $W$ obtained in this way is still a limit of smooth $k$-gonal curve, it is sufficient that the singular points of each $F_{i}$, say $R_{i}$ and $S_{i}$ (with $R_{i}$ on $Z$ and $S_{i}$ on $E_{i}$ ), are such that $2 R_{i}$ is linearly equivalent to $2 S_{i}$ on $F_{i}$ and that the points $R_{i}$ are ramification points of a $g_{k}^{1}$ on $Z$. It is possible to satisfy the last condition by Riemann-Hurwitz because $4 k-4 \geq g$. It is the torsion condition on $R_{i}-S_{i}$ which assures us that there is a $2: 1$ morphism $F_{i} \in \mathbf{P}^{1}$ ramified over $R_{i}$ and $S_{i}$. It is this torsion condition which makes (in general) false the Brill-Noether bound for $W$ (see [W], Remarks 1.12 and 2.9, [EH1], Lemma 7.3, [EH3], Theorem 1.1, [EH4], Proposition 5.2 and Corollary 5.3). To prove our claim (and hence 0.1), look at the proofs in [EH1] in which the case of a curve with general moduli is proved. To get the upper bound $\rho(g, r, d)$ for $\operatorname{dim}\left(W_{d}^{r}\right)$ for the genus $g$ starting from the bound $\rho(2 k-2, r, d)$ for the genus $2 k-2$, it would be sufficient to perform the numerical calculations in the first half of page 134 of [EH1]. To make those calculations the only missing step in our situation is part (iii) of Lemma 7 of [EH1]. To have our weaker upper bound it is sufficient to have, for each of the $s$ elliptic tails, instead of part (iii) of Lemma 7 of [EH1] which says 
"... for more than one value of the index called $i$ in [EH1] only if there are two or more independent sections of $V_{F_{i}}$ vanishing only at the two points $R_{i}$ and $S_{i}$ ", the weaker statement " $\ldots$ for more than two values of $i$ only if there are three or more independent sections of $V_{F_{i}}$ vanishing only at the two points $R_{i}$ and $S_{i}$ ". To prove this weaker statement it is sufficient to note that on each $F_{i}$ there is no $g_{d}^{2}$ with a positive divisor supported on $\left\{R_{i}, S_{i}\right\}$ because no degree 0 line bundle on $F_{i}$ has two linearly independent sections.

\section{REFERENCES}

[EH1] D. Eisenbud and J. Harris, On the Brill-Noether theorem, Open Problems, Proceedings Ravello Conference, Lecture Notes in Math., vol. 997, Springer-Verlag, Berlin and New York, 1983, pp. 131-137. MR 85g:14031

[EH2] _ Limit linear series: Basic theory, Invent. Math. 85 (1986) 337-371. MR 87k:14024

[EH3] _ The Kodaira dimension for the moduli space of curves of genus $\geq 23$, Invent. Math. 90 (1987), 359-387. MR 88g:14027

[EH4] _ Existence, decomposition, and limits of certain Weierstrass points, Invent. Math. 87 (1987), 495-515. MR 88a:14028b

$[\mathrm{HM}]$ J. Harris and D. Mumford, On the Kodaira dimension of the moduli space of curves, Invent. Math. 67 (1982), 23-86. MR 83i:14018

[W] G. Welters, A theorem of Gieseker-Petri type for Prym varieties, Ann. Sci. École Norm. Sup. (4) 18 (1985), 671-683. MR 88a:14034

Department of Mathematics, University of Trento, 38050 Povo, Trento, Italy

E-mail address: ballico@itncisca.bitnet or ballico@science.unitn.it

Department of Mathematics, Seoul National University, Seoul 151-742, Korea

E-mail address: ckeem@krsnuccl.bitnet or ckeem@math.snu.ac.kr 